\title{
2302. Research on vibration suppression of wind turbine blade based on bamboo wall three-layer damping structure
}

\author{
Jie Meng ${ }^{1}$, Dagang Sun ${ }^{2}$ \\ Taiyuan University of Science and Technology, Taiyuan, China \\ ${ }^{1,2}$ Corresponding author \\ E-mail: ${ }^{1}$ mengturbo@163.com, ${ }^{2}$ sundgbox@sina.com \\ Received 7 July 2016; received in revised form 7 December 2016; accepted 8 December 2016 \\ DOI https://doi.org/10.21595/jve.2016.17378
}

Check for updates

\begin{abstract}
As wind turbine blades tend to be long and narrow, the demand for its properties of bending resistance and vibration suppression is improving day by day. Mimicking the three-layer structure (the outer skin, the middle, the inner skin) of bamboo, this paper puts forward a new damping structure with the blade as a basic layer, one transitional layer and one free damping layer added to it. The formula of the structural loss factor is deduced by vibration differential equations. Then combined with the formula, the models of airfoil flutter and damping vibration suppression and related data, the numerical simulation is done using Matlab/Simulink Software. Consequently, the comparison diagrams of swing and wave velocity responses and their displacement responses between conditions with and without the new damping structure are gained, which indicates that the property of vibration suppression of wind turbine blades with the new damping structure is significantly improved.
\end{abstract}

Keywords: wind turbine blade structure, property of vibration suppression, bionic three-layer bamboo structure, structural loss factor.

\section{Nomenclature}

$\omega \quad$ Angular velocity

$m \quad$ Mass

$\bar{B} \quad$ Composite bending stiffness

$B \quad$ Bending stiffness

$\varepsilon \quad$ Elongation

$M \quad$ Bending moment

$v \quad$ Transverse vibration velocity

$\theta \quad$ Bending angle displacement

$P \quad$ Transverse force

$\sigma \quad$ Normal tensile and compressive stress

H Thickness

$\zeta \quad$ Distance between the center line of the barycenter of the damping beam and the basic layer surface

$\eta \quad$ Loss factor of damping structure

$\beta \quad$ Loss factor of damping material

E Elastic modulus

$x \quad$ Displacement of blade section

$\theta_{1} \quad$ Torsional angle of blade section

$k \quad$ Spring stiffness

$e \quad$ Distance between mass $G$ and torsion $E$

$\delta \quad$ Distance between aerodynamic center $A$ and mass $G$

$M_{1} \quad$ Torque

$J \quad$ Airfoil moment of inertia

I Polar moment of inertia 


$\begin{array}{ll}F & \text { Aerodynamic force } \\ r & \text { Distance between airfoil cross section and axis of rotation } \\ c & \text { Airfoil chord length } \\ w & \text { Blade cross section relative velocity } \\ \rho & \text { Air density } \\ K_{S} & \text { Structural stiffness } \\ C_{s} & \text { Structural damping } \\ K_{A} & \text { Aerodynamic stiffness } \\ C_{A} & \text { Aerodynamic damping } \\ \lambda & \text { Poisson ratio } \\ \Omega & \text { Blade wheel rotational velocity } \\ \varphi & \text { Inflow angle } \\ v_{0} & \text { Velocity in wind wheel plane } \\ u_{\infty} & \text { Wind velocity } \\ C_{l} & \text { Lift coefficient } \\ C_{d} & \text { Drag coefficient } \\ \alpha & \text { Attack angle } \\ \gamma & \text { Setting angle } \\ a & \text { Axial inducible factor } \\ a^{\prime} & \text { Tangential inducible factor }\end{array}$

\section{Introduction}

The wind turbine has long and narrow blades which vibrate under the influence of aerodynamic load, elastic force and inertial force. The most common and pernicious vibrations for large flexible blades are the aero-elastic coupling flutter and stall flutter [1]. Vibrations not only affect output power but also cause fatigue cracks and even damages on blades. Related scholars have made so many researches on the vibration phenomenon of wind turbine blades. Dossing proposes that the tuned vibration reducer is used to decrease vibration, which is difficult to implement. Murtagh, etc. raise that blades passive control is realized by the tuned mass damper to decrease the excitation force from the dynamic unbalance, but the tuned mass damper has poor adaptability. Thomas, etc. design an intelligent blade structure with variable trailing edges, but limited by the surface change rate of blade trailing edges, the effect of vibration suppression is not ideal. [2-4] John Montesanto [5], Stanciu Mariana Domnica, etc. [6]. set up a physical multi-scale progressive damage model to forecast the durability of blade structure and identify the risk areas of blade composite structures for material loading. They also studied the subcritical damage evolution and stiffness degradation of blade structures with finite-element analysis using computational micromechanics subroutines in continuum damage mechanics coupling framework to improve blade structure and lower production cost. It faces great innovations to improve blade structures, processing materials and modes $[7,8]$.

Bamboo possesses unique functional gradient structures to resist wind attacks. Domestic and foreign scholars have made so many researches on bamboo structures. Ma Jianfeng [9], etc. compare the anti-bending properties of the bionic bamboo structure with the traditional cylindrical shell by finite element simulation analysis. They draw a conclusion that the carrying capacity of the bionic bamboo structure is 1.25 times that of the traditional structure; T. Tan [10], etc. put forward a series of multi-scale experiments and numerical models to analyze the functional gradient structures of bamboo, and apply Yang modulus variation finite element model to analyze the mechanical property of bamboo in a fracture toughness experiment. Meisam K. Habibi [11], etc. study the asymmetric bending response of bamboo under different loading conditions by multiscale mechanical characteristics with a scanning electron microscope. The results show that bamboo fibers along the wall thickness of gradient distribution mainly affect the bamboo 
mechanical property. So, based on the different mechanical properties of bamboo structural parts, this paper tries to apply the bamboo three-layer structure to wind turbine blades to verify whether the vibration performance of the blades with the bionic three-layer bamboo structure is significantly improved with the help of vibration differential equations, the models of airfoil flutter and damping flutter suppression and the numerical simulation.

\section{Materials and methods}

\subsection{Bionic damping structure analysis}

Bamboo internal structure is shown in Fig. 1 [12]. Bamboo wall is composed of the outer skin, the middle and the inner skin. Their mechanical properties are closely related to their fiber volume fraction. The fiber volume fraction of the outer skin is the largest, the middle is less and the inner skin is least. It is true of their longitudinal elastic modulus and tensile strength. The reasons why bamboo has a superior ability to resist wind attacks are as follows: The outer skin has high elastic modulus and tensile strength, which can bear wind load in nature as a basic layer; Playing an important role in connecting the outer skin and the inner skin, the middle can dissipate part of the energy from wind load as a transitional layer; The inner skin can also dissipate part of the energy as a free damping layer. So, bamboo three-layer structure can be mimicked and applied to the wind turbine blade.

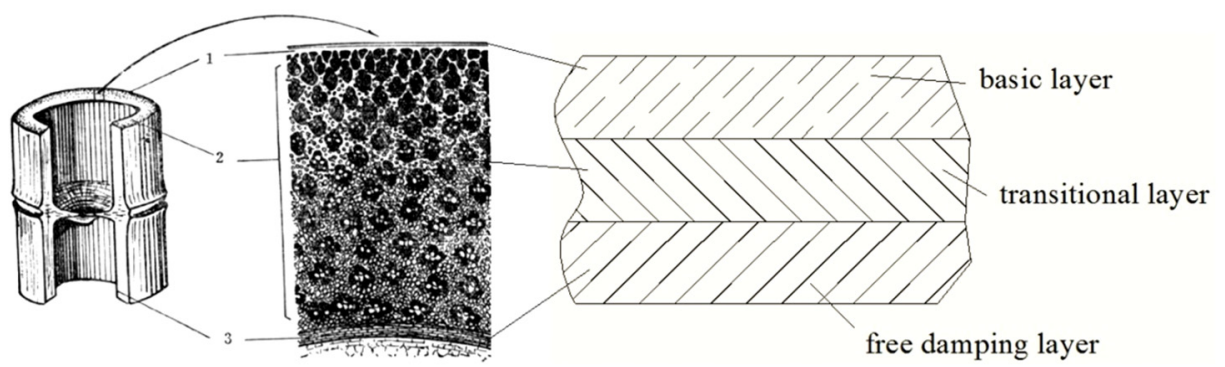

Fig. 1. Bamboo internal structure. Note: 1 - outer skin, 2 - middle, 3 - inner skin

\subsection{Model analysis of the new damping structure}

Mimicking the three-layer structure of bamboo, with the blade as a basic layer, one transitional layer and one free damping layer are added to form the new damping structure to suppress the blade vibration. The new damping structure is shown in Fig. 2.

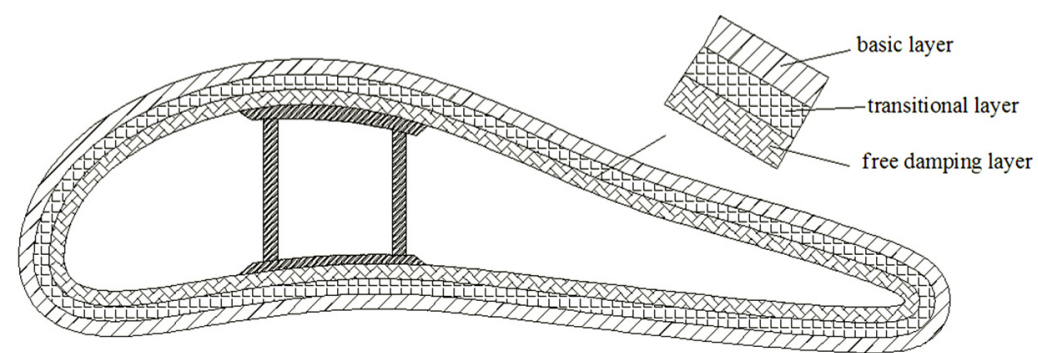

Fig. 2. New damping structure

Bamboo wall thickness is far less than its length, and blade shell thickness is also far less than its wingspan length. So, the blade structure can be abstracted as a beam and its bending vibration theory can be adopted to analyze the blade dynamics performance. Fig. 3 is the bending vibration parameter specification for the new damping beam [13]. 

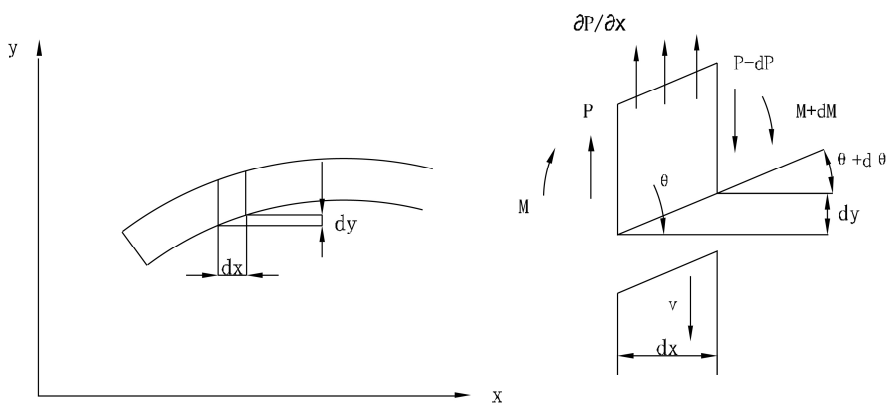

Fig. 3. Bending vibration parameter specification for the new damping beam. Note: $v$ is transverse vibration velocity; $\theta$ is bending angle displacement; $P$ is transverse force; $M$ is bending moment

In order to determine the composite bending stiffness $\bar{B}$ of the new damping beam, the center line of the barycenter should be determined first (as is shown in Fig. 4).

When the damping structural beam is bending, the elongation $\varepsilon$ of the basic, transitional and free damping layers along the longitudinal direction varies with $y$ in a linear fashion:

$\varepsilon=\frac{y d \theta}{d x}=\frac{y}{i \omega} \frac{\partial \omega}{\partial x}$

The normal tensile and compressive stress in $X$ direction is as follows:

$\sigma_{n}=E_{n} \varepsilon_{n}$

where $n=1,2,3$ represent the basic layer, the transitional layer and the free damping layer respectively. The following formula can be gained by plugging Eq. (1) into (2):

$\sigma_{n}=\frac{E_{n}}{i \omega} y \frac{\partial \omega}{\partial x}$

Under pure bending, the force acting on the beam cross section along $X$ direction is equal to zero, that is:

$\int_{-\left(H_{1}-\zeta\right)}^{H_{2}+H_{3}+\zeta} \sigma d y=0$.

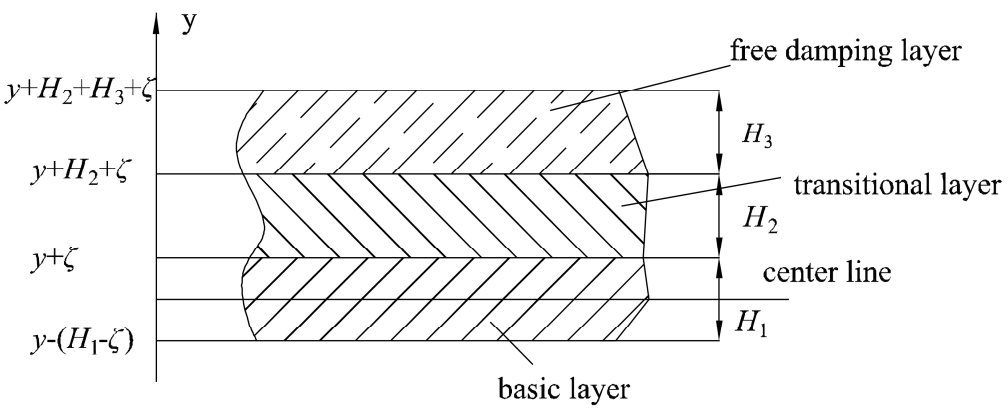

Fig. 4. Cross-section of the new damping structure: $H_{1}, H_{2}, H_{3}$ are the thickness of the basic layer, transitional layer and the free damping layer, and $\zeta$ is the distance between the center line of the barycenter of the damping beam and the basic layer surface

The curvature $\frac{1}{i \omega} \cdot \frac{\partial \omega}{\partial x}$ is a constant. If the above formula is equal to zero, then: 
$\int_{-\left(H_{1}-\zeta\right)}^{H_{2}+H_{3}+\zeta} E_{n} y d y=0$.

By Eq. (2), $\zeta$ can be expressed as:

$\zeta=\frac{1}{2} \frac{E_{1} H_{1}^{2}-E_{2} H_{2}^{2}-E_{3} H_{3}^{2}-E_{3} H_{2} H_{3}}{E_{1} H_{1}+E_{2} H_{2}+E_{3} H_{3}}$

After $\zeta$ is determined, bending moment $M$ can be expressed as:

$M=\int_{-\left(H_{1}-\zeta\right)}^{H_{2}+H_{3}+\zeta} y \sigma d y$.

Plugging formula $M=\frac{\bar{B}}{i \omega} \cdot \frac{\partial \omega}{\partial x}$ and Eq. (3) into (7), the following can be gained:

$$
\begin{aligned}
\bar{B} & =\int_{-\left(H_{1}-\zeta\right)}^{H_{2}+H_{3}+\zeta} E_{n} y^{2} d y \\
& =\frac{E_{1} H_{1}^{3}}{3}\left[\bar{e}_{2}\left(3 h_{1}^{2} h_{2}+h_{2}^{3}+3 h_{2} \frac{\zeta^{2}}{H_{1}^{2}}+3 h_{1} h_{2}^{2}+6 h_{1} h_{2} \frac{\zeta}{H_{1}}+3 h_{2}^{2} \frac{\zeta}{H_{1}}\right)\right. \\
& \left.+\bar{e}_{1}\left(h_{1}^{3}+3 h_{1}^{2} \frac{\zeta}{H_{1}}+3 h_{1} \frac{\zeta^{2}}{H_{1}^{2}}\right)+1-3 \frac{\zeta}{H_{1}}+3 \frac{\zeta^{2}}{H_{1}^{2}}\right]
\end{aligned}
$$

where $\bar{e}_{1}=\bar{E}_{2} / E_{1}, \bar{e}_{2}=\bar{E}_{3} / E_{1}, h_{1}=H_{2} / H_{1}, h_{2}=H_{3} / H_{1}$.

Eq. (6) can also be expressed as:

$\zeta=\frac{H_{1}-\bar{e}_{1} h_{1} H_{2}-\bar{e}_{2} h_{2}\left(H_{3}+H_{2}\right)}{2\left(1+\bar{e}_{1} h_{1}+\bar{e}_{2} h_{2}\right)}$.

Plugging Eq. (9) into (8), the following can be gained:

$$
\begin{aligned}
\bar{B} & =\frac{B_{1}}{1+\bar{e}_{1} h_{1}+\bar{e}_{2} h_{2}}\left[1+2 \bar{e}_{1}\left(2 h_{1}+3 h_{1}^{2}+2 h_{1}^{3}\right)+2 \bar{e}_{2}\left(2 h_{2}+6 h_{1}^{2} h_{2}+2 h_{2}^{3}+6 h_{1} h_{2}^{2}\right.\right. \\
& \left.\left.+6 h_{1} h_{2}+3 h_{2}^{2}\right)+4 \bar{e}_{1}^{2} h_{1}^{4}+12 \bar{e}_{2}^{2}\left(h_{1}^{2} h_{2}^{2}+h_{1} h_{2}^{3}\right)+4 \bar{e}_{1} \bar{e}_{2}\left(4 h_{1}^{3} h_{2}+h_{1} h_{2}^{3}+h_{2}^{4}+3 h_{1}^{2} h_{2}^{2}\right)\right],
\end{aligned}
$$

where $\bar{B}$ is the composite bending stiffness of the damping structural beam. $B_{1}$ is the bending stiffness of the ordinary beam.

Compared with the loss factor $\beta_{2}$ and $\beta_{3}$ of the viscoelastic damping materials on the transitional layer and free damping layer, the loss factor $\beta_{1}$ of the basic layer is so small that it can be ignored. Also, $\bar{B}=B(1+i \eta), \bar{e}_{1}=e\left(1+i \beta_{2}\right), \bar{e}_{2}=e\left(1+i \beta_{3}\right)$, and $\eta$ is the loss factor of the new damping structure. So, the following can be gained:

$$
\begin{aligned}
& \frac{B}{B_{1}}(1+i \eta)=\frac{1}{1+e_{1}\left(1+i \beta_{2}\right) h_{1}+e_{2}\left(1+i \beta_{3}\right) h_{2}}\left[1+2 e_{1}\left(1+i \beta_{2}\right) a+2 e_{2}\left(1+i \beta_{3}\right) b\right. \\
& \left.\quad+4 e_{1}^{2}\left(1+i \beta_{2}\right)^{2} h_{1}^{4}+12 e_{2}^{2}\left(1+i \beta_{3}\right)^{2} c+4 e_{1}\left(1+i \beta_{2}\right) e_{2}\left(1+i \beta_{3}\right) d\right],
\end{aligned}
$$

where $a, b, c$ and $d$ :

$a=2 h_{1}+3 h_{1}^{2}+2 h_{1}^{3}, \quad b=2 h_{2}+6 h_{1}^{2} h_{2}+2 h_{2}^{3}+6 h_{1} h_{2}^{2}+6 h_{1} h_{2}+3 h_{2}^{2}$,

$c=h_{1}^{2} h_{2}^{2}+h_{1} h_{2}^{3}, \quad d=4 h_{1}^{3} h_{2}+h_{1} h_{2}^{3}+h_{2}^{4}+3 h_{1}^{2} h_{2}^{2}$. 
Omitting $\beta_{2} \beta_{3}, \beta_{2}^{2}$ and $\beta_{2}^{2}$, the real component can be expressed as:

$$
\begin{aligned}
\frac{B}{B_{1}} & =\frac{1}{\left(1+e_{\left.`_{1} h_{1}+e_{2} h_{2}\right)^{2}}\right.}\left[1+e_{1}(2 a+1)+e_{2}(2 b+1)+2 e_{1}^{2}\left(2 h_{1}^{4}+a+2 e_{1} h_{1}^{4}\right)\right. \\
& +2 e_{2}^{2}\left(6 c+b+6 e_{2} c+2 e_{1} e_{2}\left(2 d+a+b+2 e_{2} d+6 e_{2} c\right)+4 e_{1}^{2} e_{2}\left(1+\beta_{2} d+h_{1}^{4}\right)\right] .
\end{aligned}
$$

Omitting $\beta_{2} \beta_{3}, \beta_{2}^{2}$ and $\beta_{2}^{2}$, the imaginary component can be expressed as:

$$
\begin{aligned}
& i \frac{B}{B_{1}} \eta=\frac{i}{\left(1+e_{1} h_{1}+e_{2} h_{2}\right)^{2}}\left\{e_{1} \beta_{2}(2 a-1)+e_{2} \beta_{3}(2 b-1)+36 e_{2}^{2} \beta_{3} c+12 e_{1}^{2} h_{1}^{4} \beta_{2}\right. \\
& +2 e_{1} e_{2}\left[2\left(\beta_{2}+\beta_{3}\right) d+(a-b)\left(\beta_{2}-\beta_{3}\right)\right]+4 e_{1}^{2} e_{2}\left[\beta_{3} d+h_{1}^{4}\left(2 \beta_{2}-\beta_{3}\right)\right] \\
& \left.\quad+4 e_{1} e_{2}^{2}\left(6 \beta_{3} c+\beta_{2} d-3 \beta_{2} c\right)\right\} .
\end{aligned}
$$

Plugging Eq. (12) into (13) and omitting the higher-order item, the formula for $\eta$ can be expressed as:

$\eta=\frac{e_{1} \beta_{2}(2 a-1)+e_{2} \beta_{3}(2 b-1)}{1+e_{1}(2 a+1)+e_{2}(2 b+1)}$.

From Eqs. (12) and (14), if the material elastic modulus of the new damping structure $\left(e_{1}=E_{2} / E_{1}\right.$ and $\left.e_{2}=E_{3} / E_{1}\right)$, the thickness ratios $\left(h_{1}=H_{2} / H_{1}\right.$ and $\left.h_{2}=H_{3} / H_{1}\right)$, the loss factors $\left(\beta_{2}\right.$ and $\left.\beta_{3}\right)$, the bending stiffness of the basic layer without the damping structure $\left(B_{1}\right)$ are known, the composite bending stiffness of the new damping structure $(B)$ and the loss factor $\eta$ can be calculated.

\subsection{Models of airfoil flutter and damping vibration suppression}

This study sets up the two degrees of freedom airfoil vibration dynamic model based on typical sections. Fig. 5 is the two degrees of freedom airfoil model and its coordinates.

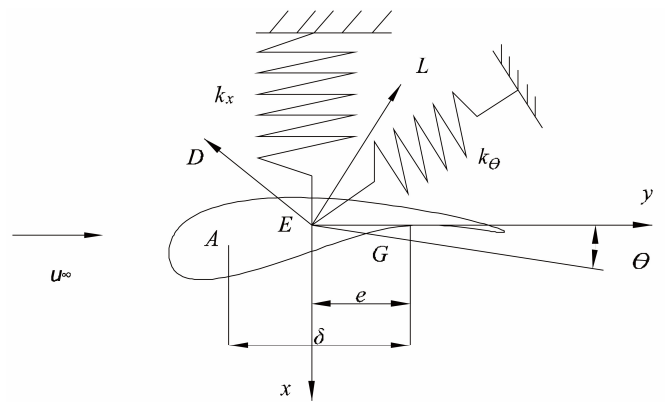

Fig. 5. Airfoil elastic supporting structure and coordinate system. Note: $x$ stands for the vertical axis and the displacement of blade section. y stands for the horizontal axis. $\theta_{1}$ stands for the torsional angle of blade section. $L$ stands the lift force. $D$ stands for the drag force. The blade is supported by a twist spring $k_{\theta}$ and a pull-press spring $k_{x}$ at the center of torsion $E$. The center of mass is $G$.

The center of torsion is $e$. The aerodynamic center is $A$. $A$ and $G$ are $\delta$ apart

The classic vibration of blades is a sort of strong aeroelastic unstable phenomenon. Its main element is the blade torsion-bending-coupled vibration [14]. The two degrees of freedom airfoil dynamic Eq. (15) is deduced by Lagrange equation:

$\left[\begin{array}{cc}J_{G} & 0 \\ 0 & m\end{array}\right]\left\{\begin{array}{l}\ddot{\theta}_{1} \\ \ddot{x}\end{array}\right\}+\left[\begin{array}{cc}k_{\theta}+e^{2} k_{x} & -e k_{x} \\ -e k_{x} & k_{x}\end{array}\right]\left\{\begin{array}{c}\theta_{1} \\ x\end{array}\right\}=\left\{\begin{array}{c}M_{1} \\ F\end{array}\right\}$, 
where $J_{G}$ is the airfoil moment of inertia about $G, M_{1}$ is the torque acted on $G$, and $F$ is the aerodynamic force acted on $G$. $e$ is the distance between $G$ and $E . m$ is the mass of airfoil cross section. $k_{x}=3 E_{1} I_{\eta} / r^{3}$ and $k_{\theta}=E_{2} I_{p} / r$ are calculated by blade material stiffness and airfoil parameters [15]. ( $E_{1}$ and $E_{2}$ are blade material shear modulus and elastic modulus. $I_{P}$ is the airfoil polar moment of inertia relative to $G$. I $\eta$ is the airfoil polar moment of inertia relative to the centroidal axis. $r$ is the distance between the airfoil cross section and the axis of rotation).

From the aerodynamic theory:

$\left\{\begin{array}{l}M_{1}=0.5 \rho w^{2} c \delta\left(C_{l} \cos \alpha+C_{d} \sin \alpha\right) \\ F=0.5 \rho w^{2} c\left(C_{l} \cos \alpha+C_{d} \sin \alpha\right)\end{array}\right.$

where $c$ is the airfoil chord length, $w$ is the blade cross section relative velocity, and $\rho$ is the air density. Eq. (16) can be also expressed as:

$\left\{\begin{array}{c}M_{1} \\ F\end{array}\right\}=\left[\begin{array}{ll}s_{11} & s_{12} \\ s_{21} & s_{22}\end{array}\right]\left\{\begin{array}{l}\theta_{1} \\ x\end{array}\right\}-\left[\begin{array}{ll}d_{11} & d_{12} \\ d_{21} & d_{22}\end{array}\right]\left\{\begin{array}{l}\dot{\theta}_{1} \\ \dot{x}\end{array}\right\}$,

where $K_{A}=\left[\begin{array}{ll}s_{11} & s_{12} \\ s_{21} & s_{22}\end{array}\right], C_{A}=\left[\begin{array}{ll}d_{11} & d_{12} \\ d_{21} & d_{22}\end{array}\right]$ are the aerodynamic stiffness and damping of aerodynamic center.

Blades don't vibrate under steady flow. Suppose blade cross section relative velocity is $w_{0}$, attack angle is $\alpha_{0}$, and setting angle is $\gamma$ at the steady-state moment. Take the airfoil at the distance of $\mathrm{r}$ away from the blade root as the research object. The following formula is derived from the airfoil velocity at the steady-state moment:

$w_{0}=\sqrt{v_{0}^{2}+(\Omega r)^{2}\left(1+a^{\prime}\right)^{2}}$.

Among them, $v_{0}$ is velocity in wind wheel plane, $v_{0}=u_{\infty}(1+a)$, and $u_{\infty}$ is wind velocity, $a$ is axial inducible factor, $\Omega$ is blade wheel rotational velocity, $a^{\prime}$ is tangential inducible factor

When the wind velocity changes, blades vibrate, the tangential velocity increment is $-\dot{x} \sin \gamma$, and the axial velocity increment is $\dot{x} \cos \gamma$, and the torsional angle of blade section is $-\theta_{1}$, the blade cross section relative velocity is as follows:

$w=\sqrt{\left(v_{0}+\dot{x} \cos \gamma\right)^{2}+(\Omega r-\dot{x} \sin \gamma)^{2}\left(1+a^{\prime}\right)^{2}}$.

Then, the attack angle $\alpha=\alpha_{0}-\theta_{1}$, the inflow angle $\varphi=\alpha+\gamma$, and its tangent function is as follows:

$\tan \varphi=\frac{v_{0}+\dot{x} \cos \gamma}{(\Omega r-\dot{x} \sin \gamma)\left(1+a^{\prime}\right)}$

As both $x$ and $\dot{\theta}_{1}$ have no relation with aerodynamic [16], so $K_{A}=\left[\begin{array}{ll}s_{11} & 0 \\ s_{21} & 0\end{array}\right], C_{A}=\left[\begin{array}{ll}0 & d_{12} \\ 0 & d_{22}\end{array}\right]$. Plugging $K_{A}$ and $C_{A}$, the airfoil flutter equation is expressed as:

$P \ddot{X}+C \dot{X}+K X=0$,

where $P=\left[\begin{array}{cc}J_{G} & 0 \\ 0 & m\end{array}\right], C=C_{A}+C_{S}, K=K_{A}+K_{S} . K_{S}$ and $C_{S}$ are the structural stiffness and structural damping: 
$K_{S}=\left[\begin{array}{cc}k_{\theta}+e^{2} k_{x} & -e k_{x} \\ -e k_{x} & k_{x}\end{array}\right], \quad C_{S}=\left[\begin{array}{cc}c_{s 11} & c_{s 12} \\ c_{s 21} & c_{s 22}\end{array}\right], \quad K_{A}=\left[\begin{array}{ll}s_{11} & 0 \\ s_{21} & 0\end{array}\right], \quad C_{A}=\left[\begin{array}{ll}0 & d_{12} \\ 0 & d_{22}\end{array}\right]$.

where:

$$
\begin{aligned}
s_{11} & =\frac{1}{2} \delta \rho w_{0}^{2} c\left[-C_{l}^{\prime}\left(\alpha_{0}\right) \cos \alpha+C_{l}\left(\alpha_{0}\right) \sin \alpha_{0}-C_{d}^{\prime}\left(\alpha_{0}\right) \sin \alpha+C_{d}\left(\alpha_{0}\right) \cos \alpha_{0}\right], \\
s_{11} & =\frac{1}{2} \delta \rho w_{0}^{2} c\left[-C_{l}^{\prime}\left(\alpha_{0}\right) \cos \alpha+C_{l}\left(\alpha_{0}\right) \sin \alpha_{0}-C_{d}^{\prime}\left(\alpha_{0}\right) \cos \alpha+C_{d}\left(\alpha_{0}\right) \sin \alpha_{0}\right], \\
d_{12} & =\rho c \delta\left(C_{l} \cos \alpha_{0}+C_{d} \sin \alpha_{0}\right)\left[v_{0} \cos \beta-\Omega r\left(1+a^{\prime}\right)^{2} \sin \gamma\right] \\
& +\frac{1}{2} \rho \delta c\left(1+a^{\prime}\right)\left(\Omega r \cos \gamma+v_{0} \sin \gamma\right)\left[\frac{d C_{l}(\varphi-\gamma)}{d \varphi} \cos (\delta-\gamma)-C_{l}(\varphi-\gamma) \sin (\varphi-\gamma)\right. \\
& \left.+\frac{d C_{d}(\varphi-\gamma)}{d \varphi} \sin (\varphi-\gamma)+C_{d}(\varphi-\gamma) \cos (\varphi-\gamma)\right], \\
d_{22} & =\rho c \delta\left(C_{l} \cos \alpha_{0}+C_{d} \sin \alpha_{0}\right)\left[v_{0} \cos \gamma-\Omega r\left(1+a^{\prime}\right)^{2} \sin \gamma\right] \\
& +\frac{1}{2} \rho c\left(1+a^{\prime}\right)\left(\Omega r \cos \gamma+v_{0} \sin \gamma\right)\left[\frac{d C_{l}(\varphi-\gamma)}{d \varphi} \cos (\delta-\gamma)-C_{l}(\varphi-\gamma) \sin (\varphi-\gamma)\right. \\
& \left.+\frac{d C_{d}(\varphi-\gamma)}{d \varphi} \sin (\varphi-\gamma)+C_{d}(\varphi-\gamma) \cos (\varphi-\gamma)\right] .
\end{aligned}
$$

If the material is assumed to be isotropic, the equivalent complex elastic stiffness of the structure can be expressed as:

$\bar{E}_{e q}=E_{e q}^{\prime}+i E_{e q}^{\prime \prime}$

As $\bar{B}=\bar{E}_{e q} I_{z}$, the real and imaginary parts at both sides of the equation are equal. So, the following formulas can be deduced as:

$B=E_{\text {eq }}^{\prime} I_{z}$

$B \eta=E_{e q}^{\prime \prime} I_{z}$.

Among them, $I_{z}$ is the moment of inertia of the damping structure to the neutral axis. The equivalent storage modulus $E_{e q}^{\prime}$ and loss modulus $E_{e q}^{\prime \prime}$ can be calculated by Eqs. (23) and (24). So, with the damping material, the damping matrix in Eq. (21) can be expressed as:

$C_{s}=\left[\begin{array}{cc}\frac{\xi B \eta}{I_{z}} & 0 \\ 0 & \frac{B \eta}{I_{z}}\end{array}\right]$.

In the matrix, $\xi=0.5(1+\lambda)$, where $\lambda$ is Poisson ratio. The analysis of the vibration suppression property of blades with the new damping structure can be done by plugging Eqs. (25) in (21).

\section{Results and discussion}

\subsection{Numerical simulation experiment}

A $600 \mathrm{kw}$ wind turbine is selected in this simulation experiment, the blade shape of which adopts FX-77-153 aeronautics airfoil and the material is fiberglass. The main design parameters are shown in Table 1[17]. The airfoil section main design parameters are shown in Table 2 [18]. 
The lift and drag coefficient functions are obtained through polynomial fitting within the scope of the designed attack angle:

$C_{l}(\alpha)=0.1043 \alpha-0.0116$,

$C_{d}(\alpha)=0.57 \times 10^{5} \alpha^{3}+0.13 \times 10^{3} \alpha^{2}-0.23 \times 10^{3} \alpha+0.0085$

where $C_{l}$ is the lift coefficient, $C_{d}$ is the drag coefficient. The designed attack angle $\alpha=10^{\circ}$, so $C_{l}(\alpha)=1.0314, C_{d}(\alpha)=0.0248, C_{l}^{\prime}(\alpha)=0.1043, C_{d}^{\prime}(\alpha)=0.0028$. The blade section parameters at the radius of $20 \%$ to $30 \%$ from the wing root was selected as the two degrees of freedom airfoil parameters, so $r=5 \mathrm{~m}, a=0.38, a^{\prime}=0.1128$ [19] based on empirical models.

The matrixes of Eqs. (21) are calculated by the parameters in Table 1 and 2 and the related formulas:

$$
\begin{aligned}
& P=\left[\begin{array}{cc}
10.40 & 0 \\
0 & 185.83
\end{array}\right], \quad K_{A}=\left[\begin{array}{cc}
-22 & 0 \\
-130 & 0
\end{array}\right], \quad C_{A}=\left[\begin{array}{ll}
0 & 1.4 \\
0 & 8.1
\end{array}\right], \\
& K S=\left[\begin{array}{cc}
5.53 \times 10^{6} & -0.617 \times 10^{3} \\
-0.617 \times 10^{3} & 0.0115 \times 10^{6}
\end{array}\right]
\end{aligned}
$$

Material parameters for the transitional layer is as follows: $\beta_{2}=0.5, E_{2}=2.9 \times 10^{8} \mathrm{~Pa}$, $\lambda=0.32$; Material parameters for the free damping layer is as follows: $\beta_{3}=1.2$, $E_{3}=1.14 \times 10^{8} \mathrm{~Pa}, \lambda=0.5$.Without considering the temperature efficiency effect of damping materials, the structural damping $C s=\left[\begin{array}{cc}5145000 & 0 \\ 0 & 1710000\end{array}\right]$. The properties of blade vibration and damping suppression are numerically simulated based on Eq. (21) by building simulation models in software Matlab/Simulink. Based on the ONERA non-linear aerodynamic model, the numerical simulations for the ordinary blade and the blade with the new damping structure are done under four different velocities: start-up wind velocity $V_{1}=4 \mathrm{~m} / \mathrm{s}$, rated wind velocity $V_{2}=15 \mathrm{~m} / \mathrm{s}$, machine halt wind velocity $V_{3}=25 \mathrm{~m} / \mathrm{s}$ and dangerous wind velocity $V_{4}=45 \mathrm{~m} / \mathrm{s}$. Fig. 6 and 7 show the swing and wave velocity responses and displacement responses of the two blades under four different wind velocities.

Table 1. Wind turbine main design parameters

\begin{tabular}{|c|c|c|c|c|c|c|}
\hline $\begin{array}{c}\text { Designed } \\
\text { item }\end{array}$ & $\begin{array}{c}\text { Output } \\
\text { power } / \mathrm{kW}\end{array}$ & $\begin{array}{c}\text { Power } \\
\text { coefficient }\end{array}$ & $\begin{array}{c}\text { Rotor velocity } \\
/ \mathrm{rad} \cdot \mathrm{s}^{-1}\end{array}$ & $\begin{array}{c}\text { Rotor } \\
\text { diameter } / \mathrm{m}\end{array}$ & $\begin{array}{c}\text { Designed attack } \\
\text { angle } /\left(^{\circ}\right)\end{array}$ & $\begin{array}{c}\text { Setting } \\
\text { angle } /\left(^{\circ}\right)\end{array}$ \\
\hline Value & 600 & 0.4 & 7 & 40 & 10 & 1.63 \\
\hline
\end{tabular}

Table 2. Foil section main design parameters

\begin{tabular}{|c|c|c|c|c|c|}
\hline $\begin{array}{c}\text { Airfoil } \\
\text { parameters }\end{array}$ & $\begin{array}{c}\text { Chord } \\
\text { length } / \mathrm{m}\end{array}$ & $\begin{array}{c}\text { Cross section mass } \\
\text { per unit } / \mathrm{kg}\end{array}$ & $\begin{array}{c}\text { Moment of } \\
\text { inertia } / \mathrm{Kg} \cdot \mathrm{m}^{-2}\end{array}$ & $\begin{array}{c}\text { Axial moment of } \\
\text { inertia } / \mathrm{cm}^{4}\end{array}$ & $\begin{array}{c}\text { Polar moment of } \\
\text { inertia } / \mathrm{cm}^{4}\end{array}$ \\
\hline Value & 1.073 & 185.83 & 10.4 & 17364 & 673110 \\
\hline
\end{tabular}

\subsection{Analysis}

From Fig. 6 and 7, as the wind velocity increases, the ordinary blade swing and wave amplitudes are increasing, and the wave displacement increases obviously; When the start-up velocity is rising to the machine halt velocity, the blade swing and wave amplitude are increasing slowly; When it is over the machine halt velocity, the blade vibration amplitude are increasing rapidly and significantly; When it reaches to the dangerous velocity, the blade cracks and even ruptures because the vibration amplitude exceeds the permitted value. For the damping blade, As the wind velocity increases, its swing and wave amplitude are also increasing, and the wave displacement increases within a small range; When the start-up velocity is rising to the machine halt velocity, its swing and wave amplitude are also increasing slowly; When it is over the machine halt velocity, the blade vibration amplitude increasement slows down significantly; When it reaches to the dangerous velocity, the blade doesn't crack and rupture because the vibration 
amplitude is within the permitted value.

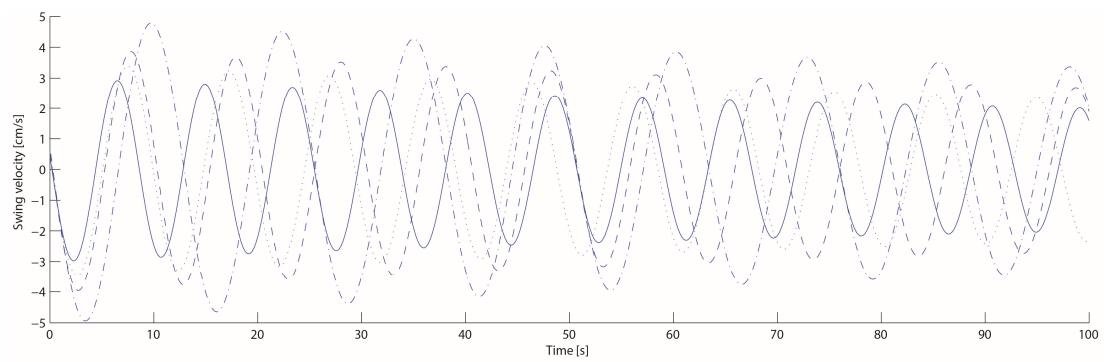

a) Ordinary blade swing velocity

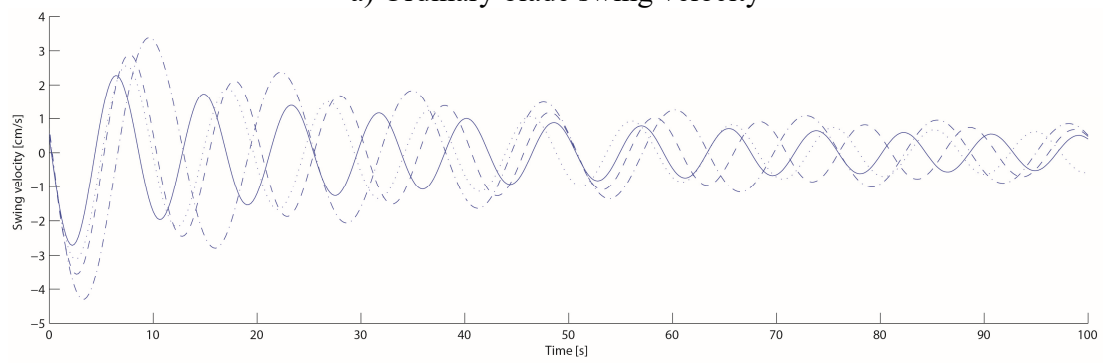

b) Damping blade swing velocity

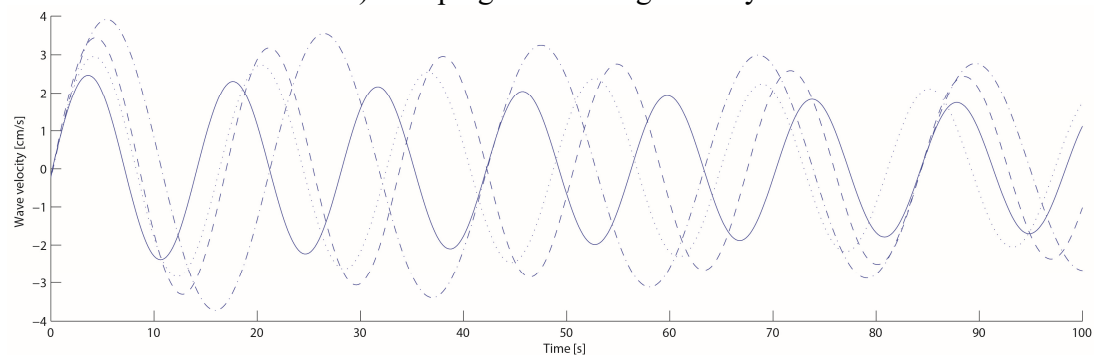

c) Ordinary blade wave velocity

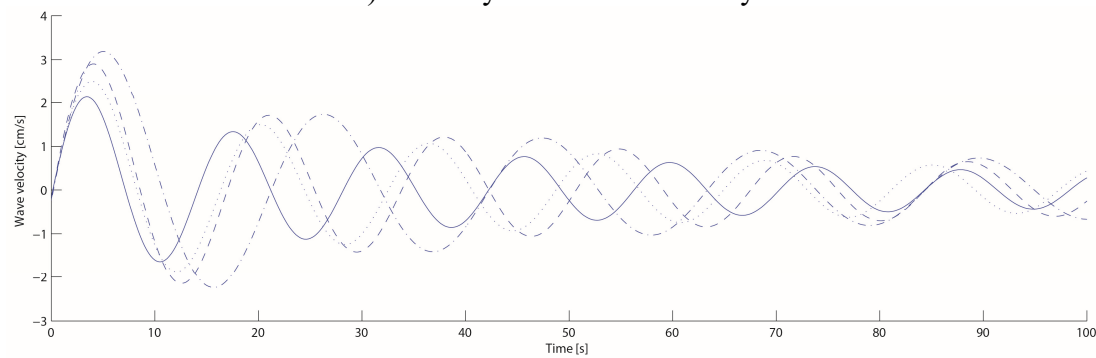

d) Damping blade wave velocity

Fig. 6. Ordinary and damping blades velocity responses. Note: compared with the ordinary blade, the standard deviation of the damping blade swing velocity decreases by $37.9 \%, 35.0 \%, 30.8 \%, 25.0 \%$ respectively; those of the wave velocity decreases by $51.1 \%, 47.9 \%, 43.7 \%$ and $37.1 \%$ respectively

Four structural loss factors $\left(\eta_{1}=0.335, \eta_{2}=0.323, \eta_{3}=0.289, \eta_{4}=0.241\right)$ of the damping blade under four different velocities are calculated by the swing and wave data. From Table 3 , compared with the ordinary blade, the standard deviation of the damping blade swing velocity decreases by $37.9 \%, 35.0 \%, 30.8 \%, 25.0 \%$ respectively; those of the swing displacement decreases by $37.9 \%, 34.8 \%, 30.8 \%$ and $25.2 \%$ respectively; those of the wave velocity decreases by $51.1 \%, 47.9 \%, 43.7 \%$ and $37.1 \%$ respectively; those of the wave displacement decreases by $51.1 \%, 48.1 \%, 43.6 \%$ and $37.1 \%$ respectively. With the transitional and free damping layers on 
the blade, the damping blade vibration amplitude is smaller than the ordinary blade because of its lower natural frequency and higher structural loss factor. The damping blade vibration amplitude decreases more quickly than the ordinary within the same period because the transitional and free damping layers turn part of the vibration energy into heat energy. Through the numerical simulation experiment, it can be concluded that the vibration amplitude of the damping blade decreases sharply within a number of vibration periods, which can delay the fatigue damage process effectively, and the property of blade vibration suppression with the new damping structure can be improved significantly.

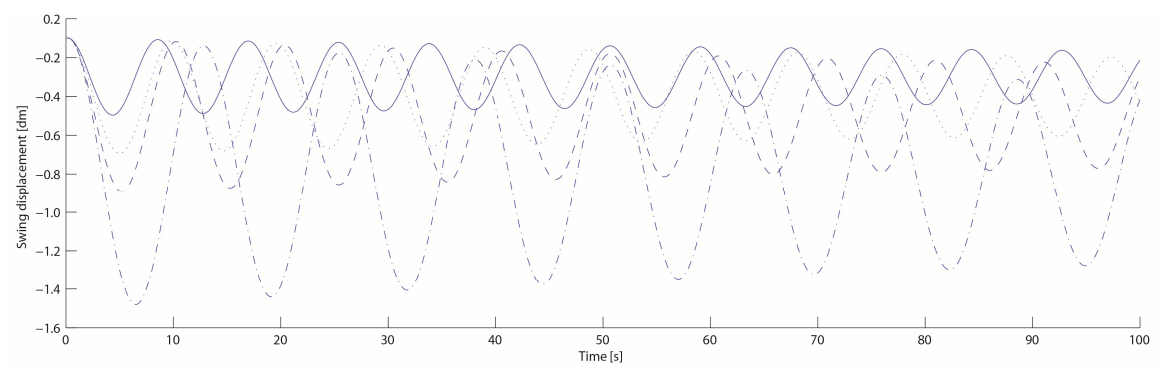

a) Ordinary blade swing displacement

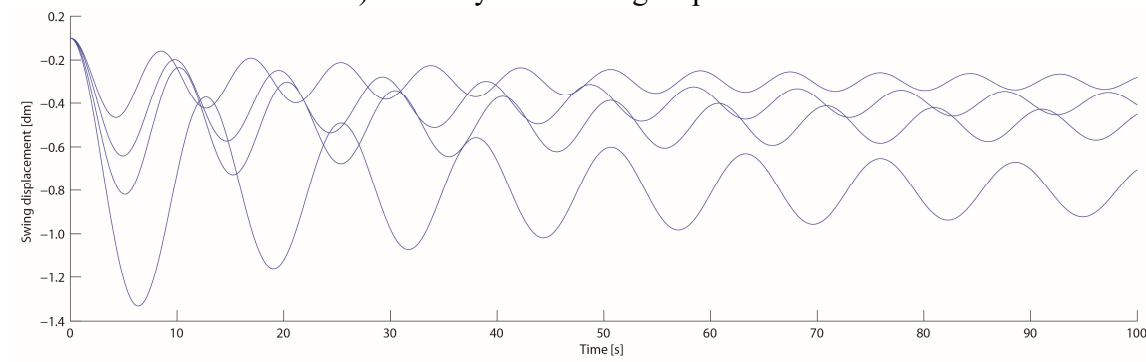

b) Damping blade swing displacement

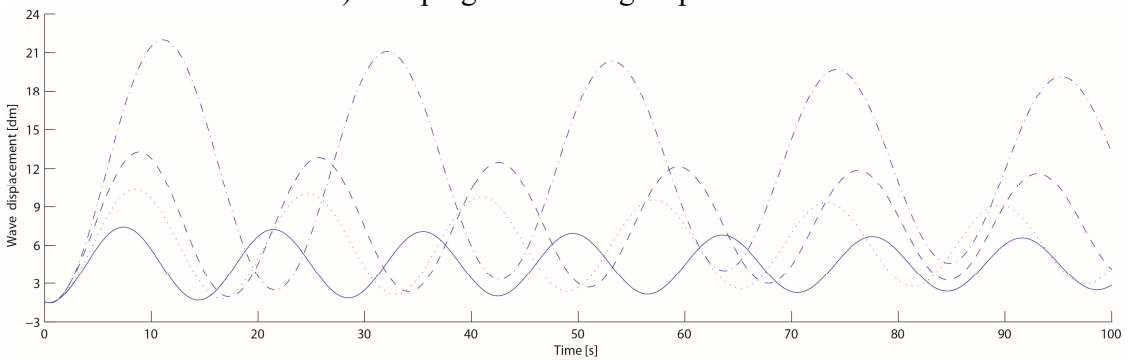

c) Ordinary blade wave displacement

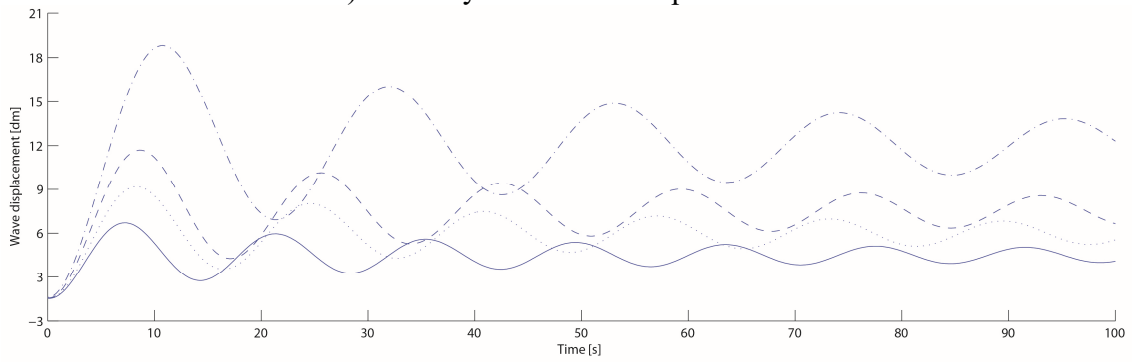

d) Damping blade wave displacement

Fig. 7. Ordinary and damping blades displacement responses. Note: compared with the ordinary blade, those of the swing displacement decreases by $37.9 \%, 34.8 \%, 30.8 \%$ and $25.2 \%$ respectively; those of the wave displacement decreases by $51.1 \%, 48.1 \%, 43.6 \%$ and $37.1 \%$ respectively 
Table 3. Simulation results

\begin{tabular}{|c|c|c|c|c|c|c|c|c|c|}
\hline \multirow{3}{*}{$\begin{array}{l}\text { Operating } \\
\text { conditions }\end{array}$} & \multirow{3}{*}{$\begin{array}{l}\text { Evaluation } \\
\text { index }\end{array}$} & \multicolumn{4}{|c|}{ Swing } & \multicolumn{4}{|c|}{ Wave } \\
\hline & & \multicolumn{2}{|c|}{ Ordinary } & \multicolumn{2}{|c|}{ Damping } & \multicolumn{2}{|c|}{ Ordinary } & \multicolumn{2}{|c|}{ Damping } \\
\hline & & $D \times 10^{-2}$ & $V \times 10^{-2}$ & $D \times 10^{-2}$ & $V \times 10^{-2}$ & $D \times 10^{-1}$ & $V \times 10^{-1}$ & $D \times 10^{-1}$ & $V \times 10^{-1}$ \\
\hline \multirow{3}{*}{ Set-up wind } & Max. & 0.00 & 0.20 & 0.00 & 0.20 & 0.60 & 0.18 & 0.60 & 0.18 \\
\hline & Min. & -0.41 & -0.21 & -0.40 & -0.20 & 0.00 & -0.19 & 0.00 & -0.17 \\
\hline & SD. & 0.09 & 0.09 & 0.07 & 0.07 & 0.17 & 0.103 & 0.14 & 0.08 \\
\hline \multirow{3}{*}{$\begin{array}{l}\text { Rated } \\
\text { wind }\end{array}$} & Max. & 0.00 & 0.23 & 0.00 & 0.22 & 0.79 & 0.21 & 0.78 & 0.20 \\
\hline & Min. & -0.54 & -0.24 & -0.53 & -0.23 & 0.00 & -0.21 & 0.00 & -0.19 \\
\hline & SD. & 0.13 & 0.18 & 0.10 & 0.09 & 0.22 & 0.12 & 0.19 & 0.09 \\
\hline \multirow{3}{*}{$\begin{array}{l}\text { Machine halt } \\
\text { wind }\end{array}$} & Max. & 0.00 & 0.28 & 0.00 & 0.27 & 1.15 & 0.25 & 1.13 & 0.25 \\
\hline & Min. & -0.78 & -0.29 & -0.77 & -0.29 & 0.00 & -0.24 & 0.00 & -0.23 \\
\hline & SD. & 0.20 & 0.15 & 0.16 & 0.11 & 0.34 & 0.15 & 0.29 & 0.13 \\
\hline \multirow{3}{*}{$\begin{array}{l}\text { Dangerous } \\
\text { wind }\end{array}$} & Max. & 0.00 & 0.37 & 0.00 & 0.36 & 2.00 & 0.34 & 1.99 & 0.33 \\
\hline & Min. & -1.36 & -0.39 & -1.34 & -0.38 & 0.00 & -0.33 & 0.00 & -0.32 \\
\hline & SD. & 0.38 & 0.21 & 0.31 & 0.17 & 0.62 & 0.21 & 0.54 & 0.18 \\
\hline
\end{tabular}

Note: $D$ stands for displacement $(\mathrm{m}) ; V$ stands for velocity $(\mathrm{m} / \mathrm{s})$. SD stands for standard deviation

\section{Conclusions}

Mimicking the three-layer bamboo structure (the outer skin, the middle, the inner skin), this paper puts forward a new structure (a basic layer, a transitional layer and a free damping layer) applied on wind turbine blades. It deduces the formula for $\zeta$ that is the distance between the center line of the barycenter of the damping beam and the basic layer surface, the formula for the composite bending stiffness of the damping beam by vibration differential equations, and the formula for structural loss factor $\eta$. Based on the models of airfoil flutter and damping vibration suppression, the numerical simulation experiment on a $600 \mathrm{kw}$ wind turbine is done using Matlab/Simulink Software. Consequently, the comparison diagrams of swing and wave velocity responses and their displacement responses between conditions with and without the new damping structure are gained. From the diagrams, as the wind velocity increases, both the ordinary blade and the damping blade swing and wave amplitudes are increasing, but the ordinary blade waving displacement increases obviously, and the damping blade increases within a small range; When the start-up velocity is rising to the machine halt velocity, both the blades swing and wave amplitude are increasing slowly; When it is over the machine halt velocity, the ordinary blade vibration amplitude are increasing rapidly and significantly, but the damping blade vibration amplitude increasement slows down significantly; When it reaches to the dangerous velocity, the ordinary blade cracks and even ruptures because its vibration amplitude exceeds the permitted value, but the damping blade doesn't crack or rupture because its vibration amplitude is within the permitted value. All the above indicate that the vibration amplitude of the damping blade decreases sharply within a number of vibration periods, which can delay the fatigue damage process effectively and the property of vibration suppression of wind turbine blades with the new damping structure is significantly improved.

\section{Acknowledgements}

The authors wish to express their sincere thanks to Liu Shizhong, Yan Bijuan, Song Yong of Damping Vibration Attenuation Laboratory of Taiyuan University of Science and Technology for their general guidance and technical advice.

\section{References}

[1] Stemple A. D., Lee S. W. A finite element model for composite beams undergoing large deflection with arbitrary cross-section warping. International Journal of Numerical Method in Engineering, Vol. 28, Issue 9, 1989, p. 2143-2160. 
[2] Dossing O. Vibration control on huge wind turbine blades using tuned absorbers. Proceeding of International Modal Analysis Conference, Vol. 1, 1997, p. 216-222.

[3] Murtagh P. J., Ghosh A., Basu B., et al. Passive control of wind turbine vibrations including blade/Tower interaction and rotationally sampled turbulence. Wind Energy, Vol. 11, Issue 4, 2008, p. 305-317.

[4] Thomas B., Gaunaa M., Christian B. Potential load reduction using airfoils with variable trailing edge geometry. Journal of Solar Energy Engineering, Vol. 127, Issue 4, 2005, p. 503-516.

[5] Montesano John, Chu Hao, Singh Chandra Veer Development of a physics-based multi-scale progressive damage model for assessing the durability of wind turbine blades. Composite Structures, Vol. 141, 2016, p. 50-62.

[6] Domnica Stanciu Mariana, Loan Curtu, Lonut Tesula Structural optimization of composite from wind turbine blades with horizontal axis using finite element analysis. Procedia Technology, Vol. 22, 2016, p. 726-733.

[7] McKenna R., v.d. Leye P. Ostman, Fichtner W. Key challenges and prospects for large wind turbines. Renewable and Sustainable Energy Reviews, Vol. 53, 2016, p. 1212-1221.

[8] Lee Kyoungsoo, Huque Ziaul, Kommalapati Raghava, Sang-Eul-Han Evaluation of equivalent structural properties of NREL phase VI wind turbine blade. Material and Manufacturing Processes, Vol. 27, Issue 27, 2016, p. 1285-1289.

[9] Ma Jian-feng, Chen Wu-yi, Zhao Ling, Zhao Da-hai Elastic buckling of bionic cylindrical shells based on bamboo. Journal of Bionic Engineering, Vol. 5, Issue 3, 2008, p. 231-238.

[10] Tan T., Rahbar N., Allameh S. M., Kwofie S., Dissmore D., Ghavami K., Soboyejo W. O. Mechanical properties of functionally graded hierarchical bamboo structures. Acta Biomaterialia, Vol. 7, 2011, p. 3796-3803.

[11] Habibi Meisam K., Samaei Arash T., Gheshlaghi Behnam, Lu Jian, Lu Yang Asymmetric flexural behavior from bamboo's functionally graded hierarchical structure: underlying mechanisms. Acta Biomaterialia, Vol. 16, 2015, p. 178-186.

[12] Yao Xishen Bamboo Culm Anatomy of China. First Edition. Science Press, Beijing, 2002.

[13] Liu Lihua Viscoelastic Damping Vibration Reduction and Noise Reduction Application Technology. First Edition. Yu Hang Press, Beijing, 1990.

[14] Hodges D. H., Dowell E. H. Nonlinear Equations of Motion for the Elastic Bending and Torsion of Twisted Nonuniform Rotor Blades. NASA TN D-7818, 1974.

[15] Dowell Earl H., Clark Robert, Cox David, et al. A Modern Course in Aeroelasticity, 4th Edition. Kluwer Academic Publisher, USA, 2004, p. 64-85.

[16] Liu Xiaoyan Analysis on Wind Turbine Blade Design and Stability. Northwestern Polytechnical University China, 2004.

[17] Chinese National Standards GB-T25383-2010, 2010.

[18] Dieter Althaus Niedrig Geschwindigkeits Profile. First Edition. Vieweg and Sohn Verlagsgesellschalt mbH, Braunschweig/Wiesbaden, 1996.

[19] Liu Xiong, Chen Yan, Ye Zhiquan Research on the aerodynamic performance prediction model for horizontal axis wind turbine. Acta Energiae Solaris Sinica, Vol. 26, Issue 6, 2005, p. 792-800.

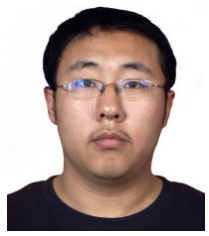

Meng Jie received the Bachelor degree of engineering in Mechanical and Electrical Engineering College of Wu Han University of Technology in Wu Han, China in 2010, then he got the Master degree of engineering in Mechanical Engineering College of Taiyuan University of Science and Technology in Taiyuan, China in 2014. He is now studying for the $\mathrm{Ph} . \mathrm{D}$. degree in Mechanical Engineering College of Taiyuan University of Science and Technology. His current research interests include vibration damping.

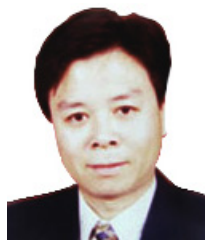

Da-gang Sun received his Ph.D. degree in College of Mechanical Science and Engineering of Jilin University in Jilin, China in 1996. Now he works in Taiyuan University of Science and Technology as a professor and in Xi'an University of Technology as a doctoral tutor. $\mathrm{He}$ is senior member of Chinese Mechanical Engineering Society, vice director of the expert committee, editor of the engineering machinery magazine. He participated in more than 20 national and provincial scientific research projects. His current research focus on mechanical vibration control and utilization and engineering machinery design theory and application research. 